\title{
二氧化碳与不饱和烃的还原羧化反应
}

\author{
张帅李雪冬何良年* \\ (元素有机化学国家重点实验室 天津化学化工协同创新中心 南开大学 天津 300071)
}

\begin{abstract}
摘要 过渡金属催化 $\mathrm{CO}_{2}$ 参与的不饱和烃还原羧化反应是合成羧酸及丙烯酸类化合物的重要途径, 具有重要的研究价 值和工业应用潜力. 过渡金属试剂与不饱和烃、 $\mathrm{CO}_{2}$ 生成稳定的金属杂环内酯或金属羧酸盐. 还原剂能够与金属杂环 内酯或金属羧酸盐发生转金属作用, 重新生成活泼催化剂, 从而实现催化剂的循环利用. 本文总结了还原剂, 包括有机 金属试剂、硅烷、嗍烷、金属粉末、甲醇和氢气等在不饱和烃与 $\mathrm{CO}_{2}$ 的还原羒化反应中的应用, 并着重描述其反应特 点和反应机理.
\end{abstract}

关键词 还原剂; 过渡金属; 不饱和烃; 还原羧化反应; 二氧化碳

\section{Reductive Carboxylation of Unsaturated Hydrocarbons with Carbon Dioxide}

\author{
Zhang, Shuai Li, Xuedong He, Liang-Nian* \\ (State Key Laboratory of Elemento-Organic Chemistry, Collaborative Innovation Center of Chemical Science and \\ Engineering, Nankai University, Tianjin 300071)
}

\begin{abstract}
Transition metal-catalyzed reductive carboxylation of unsaturated hydrocarbons with $\mathrm{CO}_{2}$ is a promising and potential strategy, offering an excellent alternative access to carboxylic acids/acrylic acids. The active transition metal species could react with unsaturated hydrocarbons and $\mathrm{CO}_{2}$ to generate the stable metallalactones or carboxylic salts. The transmetalation between reductants and metallalactones/carboxylic salts regenerates the active catalytic species. As a result, the reductive carboxylation is able to run in a catalytic mode rather than stoichiometric version. Organometal species, silanes/boranes, metal powder, methanol and hydrogens have been developed as reducing reagents in reductive carboxylation with $\mathrm{CO}_{2}$. In this perspective, the latest advances on the transition metal-catalyzed reductive carboxylation are summarized, with particular focus on the application of reductants and related reaction mechanism at a molecular level.

Keywords reducing reagents; transition metal; unsaturated hydrocarbons; reductive carboxylation; carbon dioxide
\end{abstract}

\section{1 引言}

有效利用资源和能源、保护环境, 发展绿色化学成 为大势所趋. $\mathrm{CO}_{2}$ 作为储量丰富、安全、廉价易得、特 殊的可再生资源, 通过化学转化可实现对 $\mathrm{CO}_{2}$ 的资源化 利用, 以获得高附加值的能源、材料及化工产品. $\mathrm{CO}_{2}$ “变废为宝, 高值化利用”的研究, 具有很高的科学意义 以及应用价值. 以 $\mathrm{CO}_{2}$ 为合成子, 通过构建 $\mathrm{C}-\mathrm{O}$ 键、 $\mathrm{C}$ $-\mathrm{N}$ 键和 $\mathrm{C}-\mathrm{C}$ 键等可以合成一系列高附加值产品. 然 而, $\mathrm{CO}_{2}$ 具有碳的最高氧化态 ( +4 价), 处于能量最低态. $\mathrm{CO}_{2}$ 具有热力学稳定性 $\left(\Delta_{\mathrm{f}} G=-394.38 \mathrm{~kJ} \cdot \mathrm{mol}^{-1}\right)^{[1]}$ 和动 力学惰性特点, 不利于进行化学转化. 因此, 实现 $\mathrm{CO}_{2}$ 高效转化需要消耗大量能量或使用活泼试剂.

以 $\mathrm{CO}_{2}$ 为羧源, 构建 $\mathrm{C}-\mathrm{C}$ 键的酸化反应是 $\mathrm{CO}_{2}$ 化 学转化利用的重要途径之一, 提供了一条相对于传统羧 酸制备方法(羟基或酫基的氧化反应、氰基水解反应等) 更加绿色、简洁、高效的合成路径 ${ }^{[1 ~ 3]}$. 过渡金属络合
物能够与烯、炔、共轭双烯、联烯、卤代物, 甚至活化 的碳-氢键生成金属-碳键. 随后 $\mathrm{CO}_{2}$ 通过插入到金属碳键, 构建新的碳一碳键, 生成羧酸类化合物. 这一策略 应用于以炔烃为底物合成丙烯酸及其衍生物具有广阔 的工业应用前景.

在温和条件下, 活泼的过渡金属试剂能够顺利地与 $\mathrm{CO}_{2}$ 及烯烃或联烯或炔烃反应, 生成低能态的金属杂五 元内酯 $\mathbf{A}$ 或金属羧酸盐 $\mathbf{B}$ (图 1). 内酯 $\mathbf{A}$ 和羧酸盐 $\mathbf{B}$ 化 学性质比较稳定，除非将其分解，否则很难将金属-碳 键或金属-氧键断裂. 在酸性条件下, 内酯 $\mathbf{A}$ 或金属羧 酸盐 B 经水解后可以转化为相应的羧酸产物. 例如, Saito 和Yamamoto 课题组使用当量的镍试剂及两当量的 配体与 $\mathrm{CO}_{2}$ 、炔烃形成镍杂五元环内酯, 经水解后生成 丙烯酸 ${ }^{[4,5]}$. 然而, 该方法需要消耗当量的过渡金属试 剂, 成本高, 不利于工业化应用. 在过渡金属催化二氧 化碳参与不饱和化合物的还原羧化反应中, 需要加入当 量或过量的还原剂. 还原剂能够与内酯或羒酸盐发生转

* E-mail: heln@nankai.edu.cn; Tel.: 022-23503878

Received September 26, 2015; published October 29, 2015.

Project supported by the National Natural Sciences Foundation of China (Nos. 21472103, 21421001), the Specialized Research Fund for the Doctoral Program of Higher Education (No. 20130031110013), the MOE Innovation Team (No. IRT13022) of China.

项目受国家自然科学基金(Nos. 21472103, 21421001)、教育部博士点基金(No. 20130031110013)和教育部创新才队(No. IRT13022)资助. 
金属作用重新生成活泼的过渡金属试剂，从而实现过渡 金属催化的不饱和烃还原羧化反应(图 1). 本文总结了 几类还原剂在不饱和烃还原羧化反应中的应用和反应 特点, 并着重介绍其反应机理, 希望能够对 $\mathrm{CO}_{2}$ 参与不 饱和化合物的还原羧化反应研究有所借鉴.

\section{2 还原剂在羧化反应中的转金属作用}

有机金属试剂、有机负氢化合物、稳定的金属粉末、 甲醇和氢气能够作为还原剂应用于不饱和烃的还原羧 化反应, 并表现出不同的特点和作用机理. 而且, 还原 剂能够提供氢原子或烷基，对不饱和键进行氧化加成.

\section{1 有机金属试剂}

\section{1 .1 烷基锌}

烷基锌试剂是在过渡金属催化的这类羧化反应中 使用较早、研究最深入的还原剂. 2004 年, Mori 课题 组 ${ }^{[6,7]}$ 实现了以烷基锌试剂为还原剂, 乙酰丙酮镍 $\left(\mathrm{Ni}(\mathrm{acac})_{2}\right)$ 催化的联共轭二烯的羧化反应, 生成立体选 择性的羧化环化产物(图 2). 经过系统的配体篎选, 发现 联荎类型的 $\mathrm{P}-\mathrm{O}$ 双齿配体 $(S)-\mathrm{MeO}-\mathrm{MOP}$ (图 2)具有相 当理想的效果, 可达到 $93 \%$ ee. 有机锌的结构对产物的 结构有着决定性的影响: 使用二甲基锌 $\left(\mathrm{ZnMe}_{2}\right)$ 、二苯基 锌 $\left(\mathrm{ZnPh}_{2}\right)$ 时, 得到烷基化的产物; 而使用二乙基锌 $\left(\mathrm{ZnEt}_{2}\right)$ 时，则得到非烷基化(氢原子取代)产物.

随后, Mori 课题组 ${ }^{[8]}$ 报道了以甲基锌试剂为还原 剂、镍催化的 $\mathrm{CO}_{2}$ 与炔烃还原羧化反应(图 3a). 首先, $\mathrm{Ni}(0)$ 化合物与 $\mathrm{CO}_{2}$ 、炔烃形成镍杂五元环内酯 4. 甲基
锌试剂能够将内酯 4 的镍一氧键转金属化，生成甲基镍 中间体 6. 经过还原消除过程，得到甲基化的羧化产物. 2013 年, 麻生明课题组 ${ }^{[9]}$ 报道了以乙基锌为还原剂的内 炔还原羧化反应，生成 $\beta-\mathrm{H}$ 丙烯酸类产物. 与甲基锌试 剂不同，当使用乙基锌试剂为还原剂时，乙基镍中间体 发生 $\beta-\mathrm{H}$ 消除，生成含镍-氢键的中间体. 随后经还原消 除过程，最终得到非烷基化的羧化产物.

2008 年, Rovis 课题组 ${ }^{[10]}$ 实现了 $\mathrm{Ni}(\mathrm{acac})_{2}$ 催化的苯 乙烯还原羧化反应(图 3b). 与镍催化的炔烃、联烯的还 原羧化反应不同, 此反应并没有生成镍杂五元环内 酯 ${ }^{[11]}$. $\mathrm{Ni}(\mathrm{acac})_{2}$ 经乙基锌还原形成乙基配位的镍中间体 7. 随后发生 $\beta-\mathrm{H}$ 消除, 脱去一个乙烯分子, 生成含有 镍一氢键的中间体 8 . 中间体 8 加成到苯乙烯的双键上, 得到中间体 9. 然后经过羧化过程和转金属作用, 生成 羧酸锌盐并重新生成 7 . 在 $23{ }^{\circ} \mathrm{C}$, 常压 $\mathrm{CO}_{2}$ 的条件下, 芳环上带有酯基、醚基、三氟甲基、卤素以及腈基的底 物均可转化为相应的羧酸, 而且杂环如呋喃类的烯烃也 能以较好的收率得到羧化的产物.

\section{1 .2 烷基铝}

与烷基锌类似，烷基铝也可以作为还原剂应用于不 饱和烃的还原羧化反应 ${ }^{[12 ~ 15]}$. Iwasawa 课题组 ${ }^{[13]}$ 实现了 三乙基铝 $\left(\mathrm{AlEt}_{3}\right)$ 应用于钯催化的共轭双烯或丙二烯的 还原羧化反应(催化剂为 pincer 型钯络合物(PSiP)Pd(OTf), OTf 为三氟甲磺酸根, 结构如图 4 所示), 得到 $\beta, \gamma$-不饱和酸化合物. 该反应机理与镍催化的烯烃还原 羧化反应相似. (PSiP)Pd(OTf)与三乙基铝 $\left(\mathrm{AlEt}_{3}\right)$ 发生转
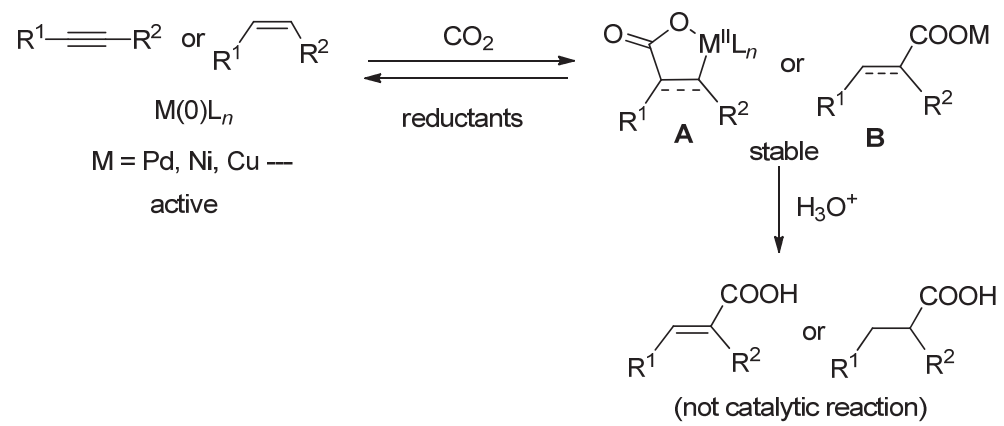

图 1 还原剂促进重新生成活泼催化剂

Figure 1 The regeneration of active catalysts promoted by reductants

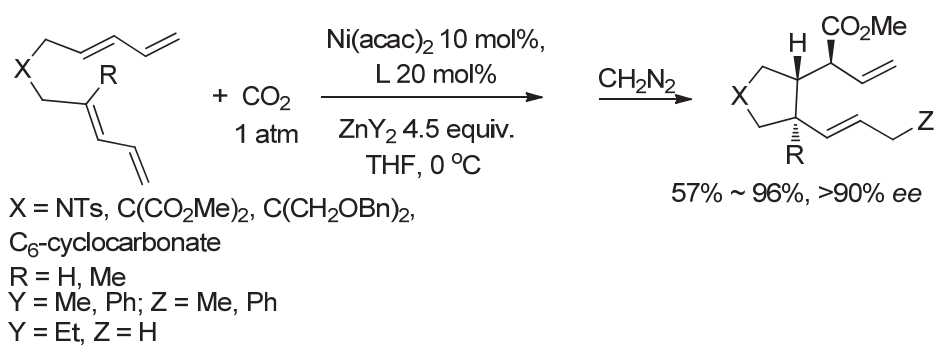

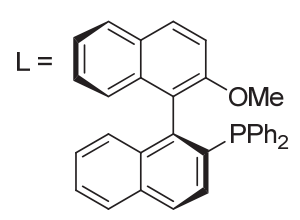

(S)-MeO-MOP

图 2 镍催化的联共轭双烯的羧化/环化反应

Figure 2 Nickel-catalyzed carboxylative cyclization of bis-1,3-dienes 
a
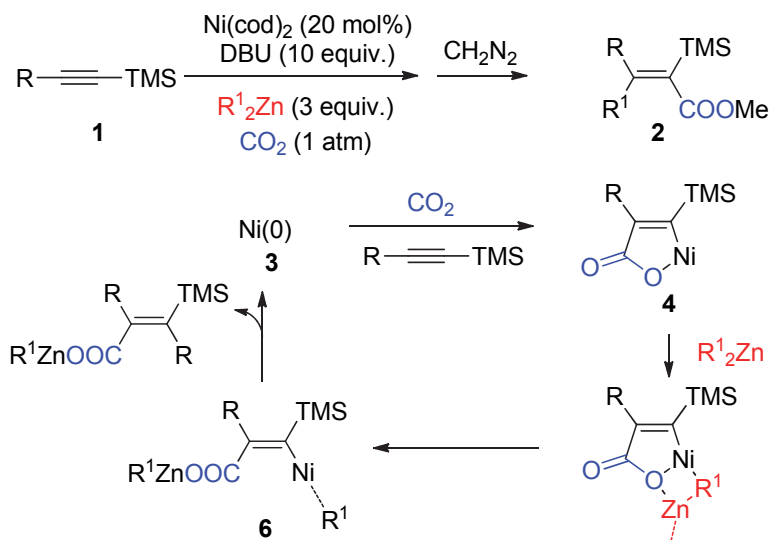
$\downarrow \mathrm{R}_{2}^{1} \mathrm{Zn}$<smiles>[R]c1c([Y6])n([R1])oc1=O</smiles>

$\mathrm{Zn}$ $\mathrm{R}^{1}$

b

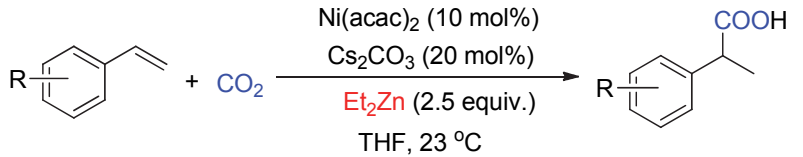

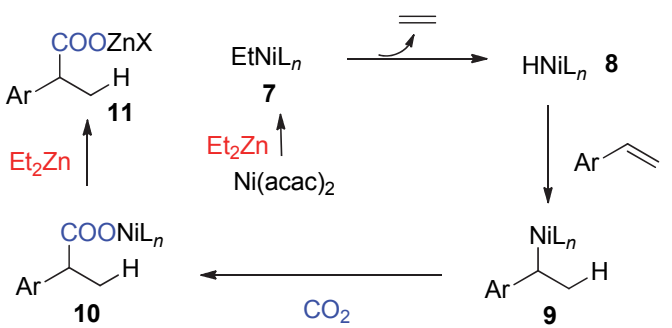

图 3 烷基锌试剂为还原剂, 镍催化的炔烃/烯烃还原羧化反应

Figure 3 Ni-catalyzed reductive carboxylation of alkyne and alkene with alkyl zinc as reductants

金属作用, 并随后发生 $\beta$ - $\mathrm{H}$ 消除反应, 生成含有钯一氢键 的活性中间体 12. 随后，该中间体对共轭双烯位阻较小 的双键进行氧化生成 $\sigma$-烯丙基络合物 13. 配体中硅基 使络合物以 $\sigma$-烯丙基络合存在，而不是以 $\pi$-烯丙基络合 物存在. 随后 $\sigma$-烯丙基钯络合物 $\gamma$ 位碳亲核进攻 $\mathrm{CO}_{2}$, 生成羧酸钯盐化合物 14. 在 $\mathrm{AlEt}_{3}$ 的作用下, 14 再次发 生转金属化及 $\beta-\mathrm{H}$ 消除反应重新生成中间体 12 , 同时得 到 $\beta, \gamma$-不饱和羧酸铝盐 15. 进一步水解便可以获得游离 的羧酸. 乙基铝试剂不仅通过转金属反应将失活的羒酸 钯盐转化为活泼的负氢钯中间体，而且可以作为氢源， 通过 $\beta-\mathrm{H}$ 消除反应将乙基上的一个氢原子转移到双键 上. 该催化体系反应条件温和(常压 $\mathrm{CO}_{2}$, 室温), 而且底 物适用性广, 带有不同取代基如醚、酯、缩酫、氨基甲 酸酯、酮等的底物能够高效地转化为 $\beta, \gamma$-不饱和䊣酸.

\subsection{3 格氏试剂}

格氏试剂是一类非常活泼的有机化合物，能够与 $\mathrm{CO}_{2}$ 迅速反应生成弮酸化合物. 2012 年, Thomas 课题 组 ${ }^{[16]}$ 报道了一例铁催化、格氏试剂为还原剂, 苯乙烯类 化合物的还原羧化反应(图 5). 在 $\mathrm{FeCl}_{2} / \mathrm{PDI}(\mathrm{PDI}=$ bis(imino)pyridine, 二亚胺基吡啶)催化下，乙基溴化镁 与苯乙烯发生转金属反应，几乎当量地转化为格氏试剂 (1-苯乙基)溴化镁 17. 随后将 $\mathrm{CO}_{2}$ 通入到反应体系中, 与所形成的格氏试剂 17 反应, 生成羧化产物 18 . 铁催 化剂的作用主要是促进格氏试剂的转移, 而对 $\mathrm{CO}_{2}$ 的插 入过程不起作用. 该反应具有较高的区域选择性, 主要 生成 $\alpha$-甲基苯乙酸类化合物. 然而, 该反应体系不能兼 容对格氏试剂敏感的官能团.

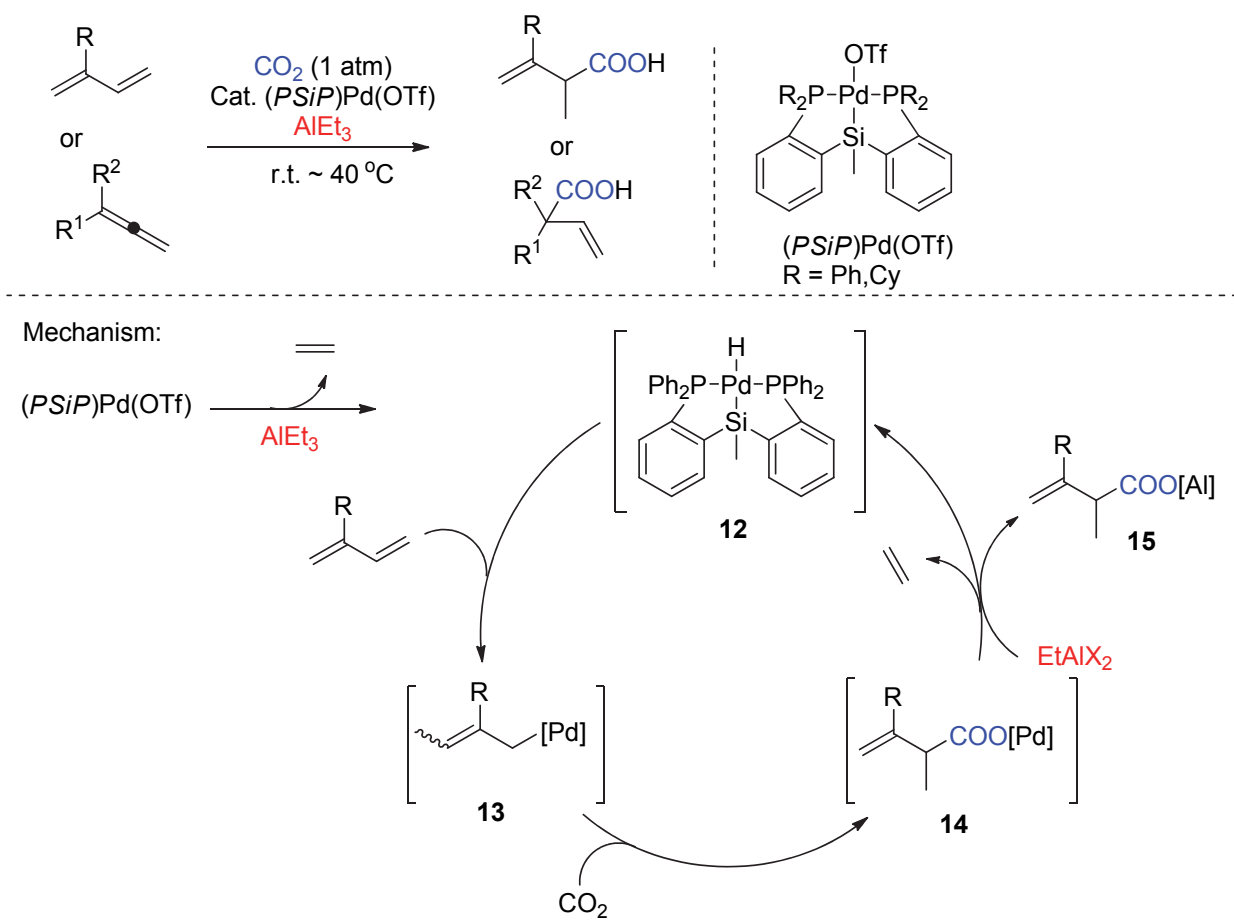

图 4 烷基铝试剂促进的丙二烯和共轭双烯的还原羧化反应

Figure 4 Pd-catalyzed hydrocarboxylation of 1,3-dienes and allenes with organo aluminum as reductants 


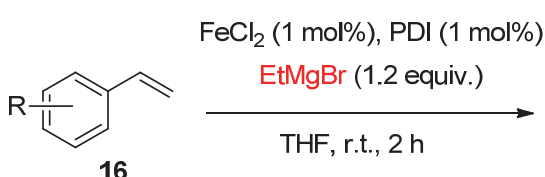

16

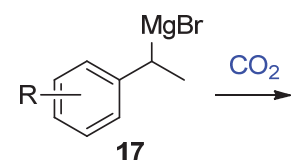

17

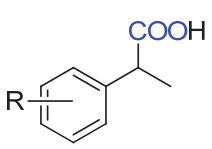

18

up to $96 \%$<smiles>CCCc1cccc(CC)c1N=C(C)c1cccc(C(C)=Nc2c(Br)cccc2P(C)c2ccccc2)n1</smiles>

图 5 格氏试剂应用于铁催化的还原羧化反应

Figure 5 Grignard reagents promoted hydrocarboxylation of styrene

以上三种有机金属试剂作为还原剂, 实现了反应的 催化循环, 极大促进了不饱和烃与 $\mathrm{CO}_{2}$ 还原羒化反应的 研究进程. 但是, 从绿色化学和 $\mathrm{CO}_{2}$ 资源化利用的角度 考虑，该策略还有一定的局限: (一)有机金属试剂对空 气、水敏感; (二)催化体系需要过量的有机金属试剂作为 还原剂, 不利于其工业化应用. 因此, 使用廉价、易操作 的还原剂是这类还原羧化反应研究的重点之一.

\section{2 硅烷/硼烷}

硅烷、硼烷是一类性质稳定、应用广泛的还原剂, 其 在不饱和烃的还原羒化反应中的应用受到了广泛关

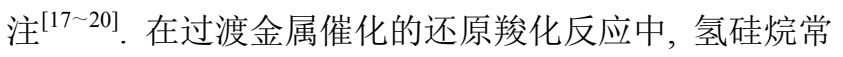
与 $\mathrm{Cu}(\mathrm{I})$ 催化剂搭配, 实现催化循环. 2011 年, Tsuji 课题 组 ${ }^{[17]}$ 报道了以氢硅烷作为还原剂, [IMesCuF] (结构如图 6 所示)催化的炔烃还原羧化反应, 生成顺式加成的丙烯 酸类化合物(图 5). 催化前体 [IMesCuF] 与氢硅烷作用, 形成由负氢取代氟的卡宾铜(I)中间体 19. 随后对炔烃 进行顺式加成, 将负氢转移到炔烃上, 生成含双键的铜 中间体 20. 中间体 20 随即与 $\mathrm{CO}_{2}$ 反应生成 $\alpha, \beta$-不饱和 酸铜盐 21. 最后, 羧酸铜盐 21 与氢硅烷发生转金属作 用, 重新生成催化剂 $\mathbf{1 9}$ 继续参与催化循环, 同时生成了 $\alpha, \beta$-不饱和酸硅酯. 酸化后即获得目标产物. 一系列烷 基或芳香基取代的对称及不对称的内炔都能以较高转 化率转化为对应的 $\alpha, \beta$-不饱和酸, 尽管反应条件略有不 同.

2012 年, 侯召民课题组 ${ }^{[20]}$ 报道了炔烃与硼烷、 $\mathrm{CO}_{2}$ 的嗍羧化反应. 在温和反应条件(常压 $\left.\mathrm{CO}_{2}, 80{ }^{\circ} \mathrm{C}\right)$ 下, 以 $N, N$-二 (2,4,6-三甲基苯基) $N$-杂环卡宾铜络合物 [(SIMes) CuCl] 为催化剂, 双联频哪醇硼酸酯 $\left[\mathrm{B}_{2}(\mathrm{pin})_{2}\right]$ 为 嗍源和还原剂, 二烷基炔、烷基/芳香基炔、苯乙炔类化 合物以高收率 $(64 \% \sim 94 \%)$ 转化为 $\alpha, \beta$-不饱和- $\beta$-硼内酯 盐(图 7). 首先, $(\mathrm{SIMes}) \mathrm{CuCl}$ 与 $\mathrm{LiO}^{t} \mathrm{Bu}$ 反应生成 SIMesCu-O ${ }^{t} \mathrm{Bu}(\mathbf{2 3})$. 在 $\mathrm{B}_{2}(\mathrm{pin})_{2}$ 的转金属作用下, 23 转 化为中间体(SIMes)Cu-B(pin) (24). 中间体 24 的铜-嗍键 对炔烃三键顺式氧化加成, 形成 $\beta$-硼基烯基铜络合物 25. 随后, $\mathrm{CO}_{2}$ 插入络合物 $\mathbf{2 5}$ 的铜一碳键并且发生环化 反应, 得到 $\beta$-嗍内酯衍生物 $\mathbf{2 6}$. 最后, 铜络合物与 $\mathrm{LiO} t \mathrm{Bu}$ 发生转金属作用, 重新生成(SIMes) Cu- ${ }^{t} \mathrm{Bu}$ (23), 并释放产物 $\alpha, \beta$-不饱和硼内酯盐. 而且, 在钯催化 剂作用下, $\alpha, \beta$-不饱和硼酯盐能够与亲核试剂(如碘苯)转 化为多官能团取代的丙烯酸类化合物(图 7). 以硅嗍烷
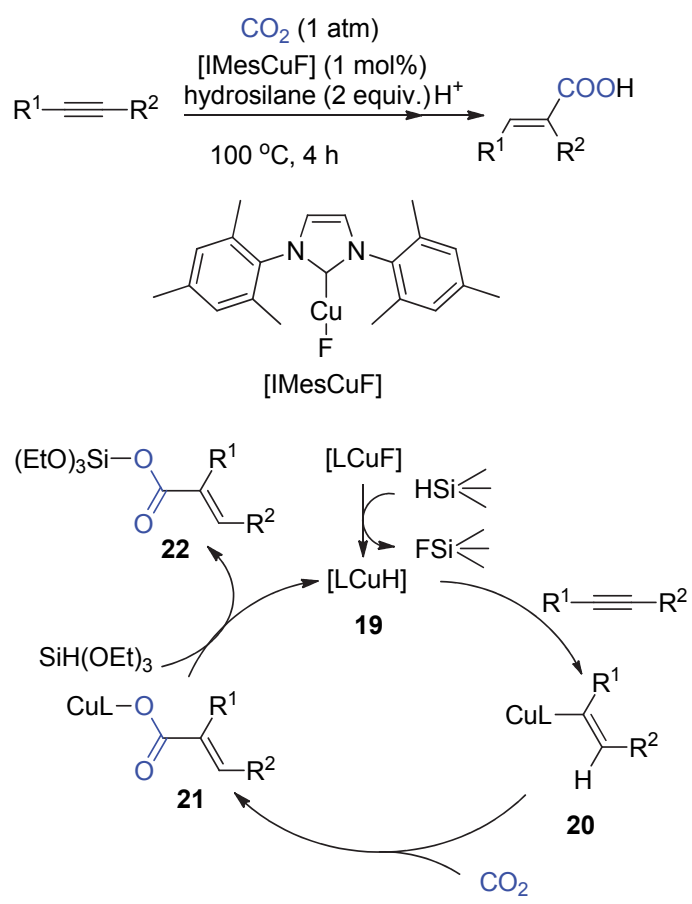

图 6 铜催化、氢硅烷参与的炔烃还原羧化反应

Figure 6 Cu-catalyzed hydrocarboxylation of alkynes with silanes
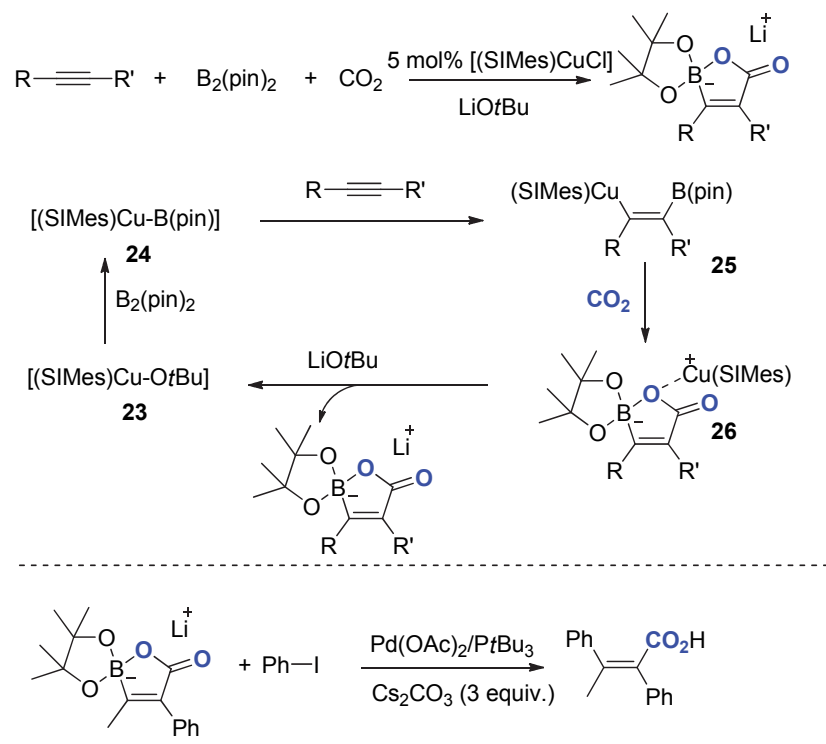

图 7 铜卡宾催化内炔、 $\mathrm{CO}_{2}$ 和硼烷的硼化/着化反应

Figure $7 \mathrm{Cu}$-catalyzed boracarboxylation of alkynes, diborane and $\mathrm{CO}_{2}$ 
为还原剂和硅源, 炔烃也能够与 $\mathrm{CO}_{2}$ 发生类似的转化反 应, 生成硅杂五元环内酯 ${ }^{[19]}$.

\section{3 金属粉末}

金属粉末作为还原剂具有性质稳定、价格低廉、操 作容易、后处理简便等优势. 近年来, 金属粉末作为还 原剂在碳卤键和碳氧键的羧化反应获得长足的发展, 已

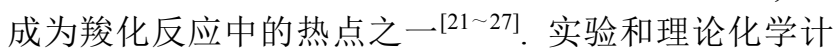
算证明, $\mathrm{CO}_{2}$ 容易插入一价镍中间体的镍碳键, 但是不 能插入二价镍络合物的镍碳键. 金属粉末对二价镍中间 体进行两次单电子还原.二价镍化合物首先被还原为一 价镍中间体, $\mathrm{CO}_{2}$ 迅速插入镍碳键生成羧酸镍盐. 随后, 生成的羧酸镍盐能够被金属粉末进一步还原为零价镍 络合物，进行下一次催化，同时生成羧酸盐.

2015 年, Martin 课题组 ${ }^{[30]}$ 报道了锰粉作为还原剂应 用于卤代炔的环化/羧化反应(图 8a). 首先, 溴化镍还原 为零价镍络合物, 插入碳溴键生成烷基镍中间体. 烷基 镍络合物随后对分子内碳碳三键顺式氧化加成, 生成烯 基镍中间体 28. 随后被锰粉还原为一价镍络合物, $\mathrm{CO}_{2}$ 插入镍(I)-碳键生成目标产物. 该反应体系对不同的反 应底物有明显的区域(顺/反)选择性. 当 $\mathrm{R}^{2}$ 取代基为氢 时，烷基和羧基加成在碳碳三键的同侧，生成产物 29; 当取代基为烷基时, 烯基镍中间体迅速发生异构化, 得 到烷基和羧基为反式结构的羧化产物 $\mathbf{3 0}$.

锌粉能够作为还原剂应用于镍催化的内炔双羧化

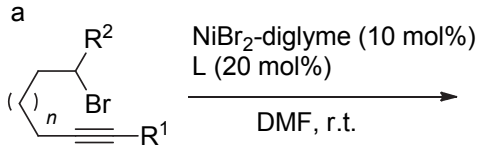

27

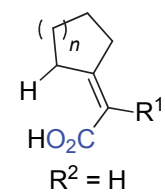

29

30 (anti)

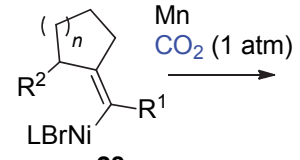

28

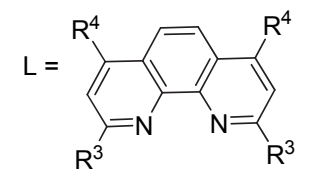

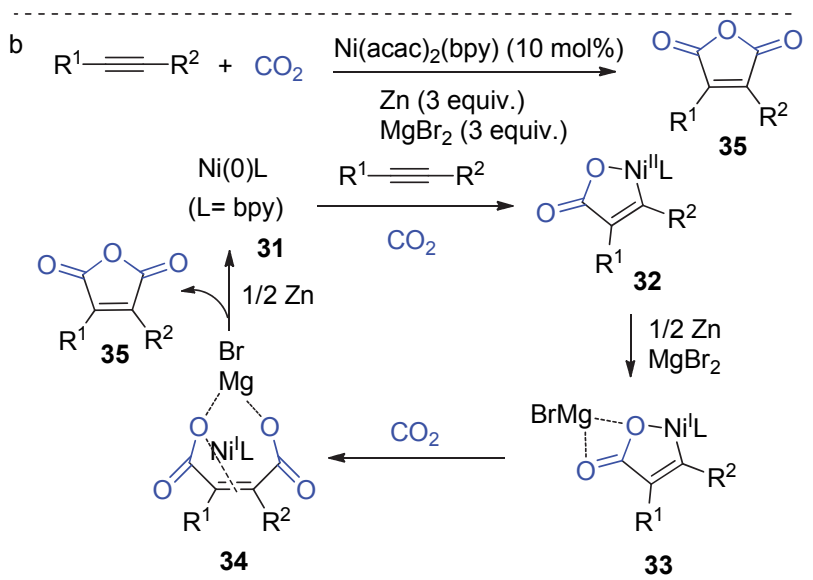

图 8 金属粉末在不饱和烃还原羧化反应中的应用

Figure 8 Metal powder as reductants in the hydrocarboxylation
反应(如图 8b) ${ }^{[31]}$. 在室温、常压 $\mathrm{CO}_{2}$ 条件下, 以锌粉为 还原剂、溴化美为添加剂, 一系列烷基取代的内炔类化 合物以高收率转化为顺丁烯羧酸䣲类产物. 通过密度泛 函理论计算, 对此双羧化反应的机理进行了详细的理论 研究. 还原剂锌粉和添加剂溴化镁的共同作用是实现此 双羧化反应的关键. 首先, 乙酰丙酮镍被锌粉还原为活 泼的催化剂 $\mathrm{Ni}^{0}$ (bpy) 31. $\mathrm{Ni}^{0}$ (bpy) 与内炔和 $\mathrm{CO}_{2}$ 发生氧化 环化反应, 形成镍(II)杂五元环内酯 32. 此为第一次羧 化反应. 与镍催化的碳卤键羧化反应相似, $\mathrm{CO}_{2}$ 不能插 入镍(II)-碳键. 因此, 二价镍中间体 $\mathbf{3 2}$ 与锌粉和溴化镁 发生单电子还原反应，生成一价镍中间体 $\mathbf{3 3}$, 才能够进 行第二次羧化反应. $\mathrm{MgBr}$ 部分能够与羧基和第二个 $\mathrm{CO}_{2}$ 相互作用, 促进 $\mathrm{CO}_{2}$ 插入镍(I)-碳键生成 34. 最后, 在锌粉的转金属作用下, 中间体 $\mathbf{3 4}$ 被锌粉进一步还原, 重新生成为零价镍催化剂 31 进行下一轮催化循环, 并 且得到双羧化产物 35 .

\section{4 氢气和甲醇}

氢气和甲醇是一类更加稳定且用途广泛的清洁还 原性试剂. 氢气或甲醇参与还原羧化反应具有环境友 好、原子经济性高等优点, 符合绿色化学和可持续发展 的要求. 氢气和甲醇作为还原剂, 在催化剂作用下可将 $\mathrm{CO}_{2}$ 还原为一氧化碳. 随后不饱和烃与一氧化碳发生羰 基化反应, 得到羧酸产物. $\mathrm{CO}_{2}$ 代替有毒、易燃的一氧化 碳进行羰基化反应，提供了一个新颖的反应策略，具有 重要的科研价值和美好的工业前景.

2013 年, Leitner 课题组 ${ }^{[32]}$ 报道了酸性体系中, 使用 氢气作为还原剂, $\left[\left\{\mathrm{RhCl}(\mathrm{CO})_{2}\right\}_{2}\right] / \mathrm{PPh}_{3}$ 催化的烯烃还原 羧化反应(图 9a). 在碘甲烷为助催化剂, $180{ }^{\circ} \mathrm{C}$ 条件下, 一系列烯烃类化合物能够高效地转化为相应的羧酸(收 率 62\% 91\%). 但是，作者并没有尝试含极性取代基的 烯烃底物. 同位素示踪实验发现, 产物羧基中的碳原子 来源于 ${ }^{13} \mathrm{CO}_{2}$. 在体系中额外加入 $\mathrm{H}_{2}{ }^{18} \mathrm{O}$ 的条件下, 产物 羧基的一个氧原子来源于 $\mathrm{H}_{2}{ }^{18} \mathrm{O}$. 以上实验证明该催化 反应包括两个催化循环: (一)在铑(I)催化剂作用下, 氢 气将 $\mathrm{CO}_{2}$ 还原为一氧化碳和水; (二) 一氧化碳参与铑催 化的羰基化反应, 随后发生水解生成羧酸产物.

2014 年, Beller 课题组 ${ }^{[33]}$ 报道了离子液体 $[\mathrm{Bmim}] \mathrm{Cl}$ 体系中 $\mathrm{Ru}_{3}(\mathrm{CO})_{12}$ 催化的烯烃还原羧化反应(图 9b). 其 中, 甲醇在反应体系中既是溶剂, 又是还原剂. 实验中 检测到一氧化碳、甲酸甲酯等还原产物, 表明甲醇在体 系中能够将 $\mathrm{CO}_{2}$ 还原为一氧化碳. 因此, 作者提出了三 条可能的反应途径. 途径一: 醇与 $\mathrm{CO}_{2}$ 作用, 先将 $\mathrm{CO}_{2}$ 还原为一氧化碳, 然后烯烃、一氧化碳与醇进行三组分 偶联, 得到饱和羧酸酯. 途径二: 醇与 $\mathrm{CO}_{2}$ 结合还原得 到甲酸酯, 甲酸酯与烯烃加成获得饱和羧酸酯, 也可能 甲酸酯分解得到一氧化碳进行途径一的历程. 途径三: 醇脱去一分子氢气, 得到醛, 醛再分解得到一氧化碳, 然后烯烃、一氧化碳与醇进行三组分偶联, 得到饱和的 
羧酸酯. 通过同位素示踪实验, 表明途径一是主要途径.

$\mathrm{H}_{2}$ as the reductant:

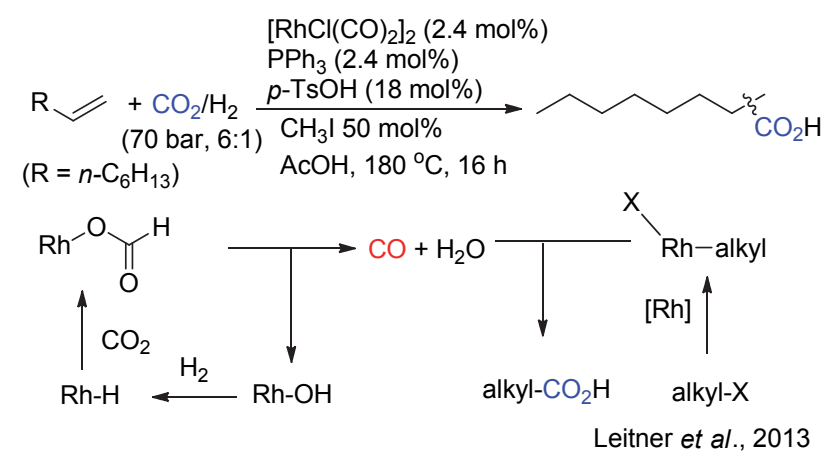

$\mathrm{CH}_{3} \mathrm{OH}$ as the reductant:

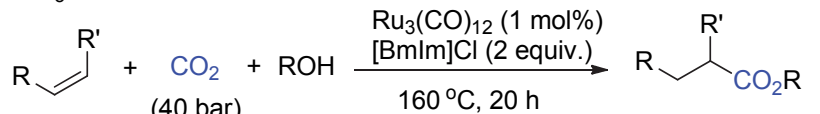

(40 bar)
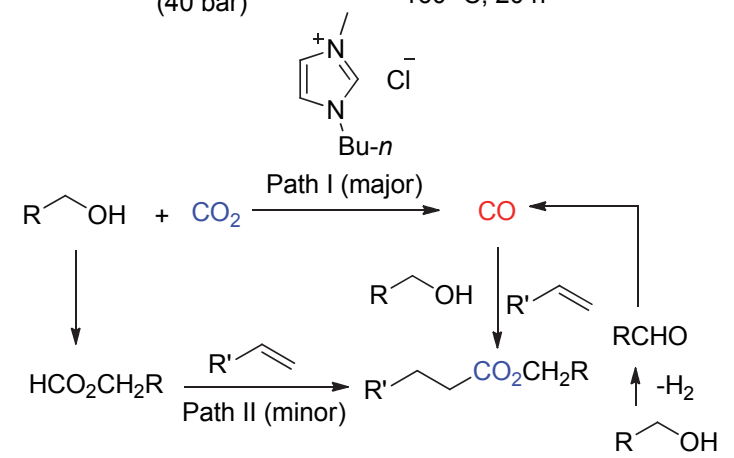

Path III (minor)

Beller et al., 2014

图 9 氢气/甲醇作为还原剂、 $\mathrm{CO}_{2}$ 作为 $\mathrm{CO}$ 类似物的羰基化反应

Figure 9 Carbonylation reactions with $\mathrm{CO}_{2}$ as $\mathrm{CO}$ surrogates, $\mathrm{H}_{2} / \mathrm{MeOH}$ as reductants

\section{3 总结}

有效利用资源和能源、保护环境, 发展绿色化学成 为大势所趋. 二氧化碳作为储量丰富、安全、特殊的可 再生资源, 通过化学转化可实现对二氧化碳的资源化利 用, 以获得高附加值的能源、材料及化工产品. 从绿色 化学与可持续发展的角度考虑, 设计二氧化碳既作为反 应介质又是一种反应物, 实现温和条件下二氧化碳的高 效转化与利用, 是当前绿色化学研究领域中最具挑战性 的研究课题之一. 二氧化碳“变废为宝, 高值化利用”的 研究, 具有很高的科学意义以及应用价值 ${ }^{[34 \sim 43]}$.

由于二氧化碳的热力学稳定性和动力学惰性, 实现 $\mathrm{CO}_{2}$ 的资源化利用需要对 $\mathrm{CO}_{2}$ 进行活化 ${ }^{[41 \sim 43]}$. 过渡金属 催化的不饱和烃与 $\mathrm{CO}_{2}$ 生成羒酸或丙烯酸类化合物的 还原羧化反应, 是一个前景广阔、充满挑战性的研究课 题. 虽然过渡金属催化 $\mathrm{CO}_{2}$ 参与不饱和化合物的还原羧 化反应的基础研究方面取得了显著进展, 但还没有实现 大规模应用, 还有待实质性的突破. 许多体系仍然需要 使用对空气和水非常敏感的还原性试剂, 底物适用性有
限. 另一方面，尽管这些反应还没有应用于大规模生产, 但在精细化工产品的生产如特定羧酸及其衍生物的制 备等方面极具潜力.

以甲醇和氢气为还原剂合成羧酸类化合物是 $\mathrm{CO}_{2}$ 高值化利用的重要策略. 从绿色化学和工业化生产的角 度考虑，该策略使用储量丰富、安全的 $\mathrm{CO}_{2}$ 代替一氧化 碳，具有广阔的工业应用前景。但是，该方法反应条件 苛刻, 而且 $\mathrm{CO}_{2}$ 还原为一氧化碳的效率较低. 因此发展 反应条件温和，催化效率更高的催化体系是该类反应工 业化应用的关键. 可以预见随着研究的不断深入, 不饱 和烃的还原羧化反应将会获得突破.

\section{作者简介}

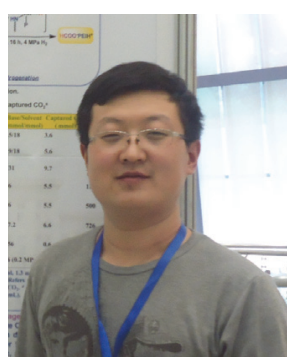

张帅, 2010 年毕业于南开大学. 2010 年至今为南开大学元 素有机化学国家重点实验室博士研究生, 师从何良年教授, 主要研究方向为“二氧化碳的化学捕集” 和“过渡金属催化 的羒化反应”.

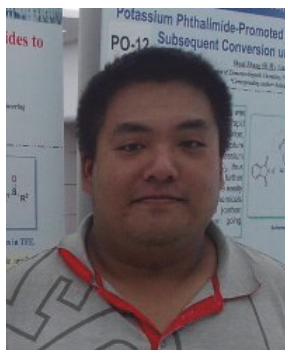

李雪冬, 2013 年毕业于南开大学. 2013 年至今为南开大学 元素有机化学国家重点实验室博士研究生, 师从何良年教授, 主要研究方向为 “过渡金属催化的 $\mathrm{CO}_{2}$ 羒化反应” 和 “过渡 金属催化的 $\mathrm{CO}_{2}$ 还原反应”

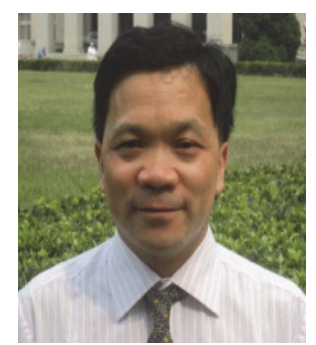

何良年, 1996 年获理学博士学位(南开大学). 1996 年至 2003 年分别在武汉大学、日本产业技术综合研究所做博士后, 从事绿色化学领域的研究. 1998 年入选湖北高等学校跨世纪 学术带头人. 2003 年回国, 被聘为南开大学教授, 2004 年 4 月 被批准为博士生导师. 2009 年楚天学者特聘教授, 2011 年英国 
皇家化学会 Fellow(FRSC). 从事二氧化碳资源化利用、绿色化 学、生物质能等领域研究, 在重要学术刊物上发表学术论文 150 多篇. 撰写英文专著 1 部、中文书籍 2 部以及英文书籍章 节 10 部, 获得授权中外专利 8 项, 受邀在国际学术会议上作 报告 50 余次. 现任 Current Organic Synthesis 主编、Journal of $\mathrm{CO}_{2}$ Utilization, Sustainable Chemical Processes 等多种国际学 术期刊编委, Springer “Green Chemistry and Sustainable Technology”丛书主编, The Advisory Board of the new book Series published by CRC Press "Green Syntheses".

\section{References}

[1] Yang, Z.-Z.; He, L.-N.; Gao, J. Energy Environ. Sci. 2012, 5, 6602.

[2] Yang, Z.-Z.; Zhao, Y.-N.; He, L.-N. RSC Adv. Rev. 2011, 1, 545.

[3] Liu, Q.; Wu, L.; Jackstell, R.; Beller, M. Nat. Commun. 2015, 6, 5933.

[4] Saito, S.; Nakagawa, S.; Koizumi, T.; Hirayama, K.; Yamamoto, Y. J. Org. Chem. 1999, 64, 3975.

[5] Takimoto, M.; Shimizu, K.; Mori, M. Org. Lett. 2001, 3, 3345.

[6] Takimoto, M.; Mori, M. J. Am. Chem. Soc. 2002, 124, 10008.

[7] Takimoto, M.; Nakamura, Y.; Kimura, K.; Mori, M. J. Am. Chem. Soc. 2004, 126, 5956.

[8] Shimizu, K.; Sato, Y.; Mori, M. Org. Lett. 2005, 7, 195.

[9] Li, S.; Yuan, W.; Ma, S. Angew. Chem., Int. Ed. 2011, 50, 2578.

[10] Williams, C. M.; Johnson, J. B.; Rovis, T. J. Am. Chem. Soc. 2008, 130, 14936.

[11] Yuan, R.; Lin, Z. Organometallics 2014, 33, 7147.

[12] Takimoto, M.; Hou, Z. Chem. Eur. J. 2013, 19, 11439.

[13] Takaya, J.; Iwasawa, N. J. Am. Chem. Soc. 2008, 130, 15254.

[14] Takaya, S. J.; Iwasawa, N. Org. Lett. 2011, 13, 1698.

[15] Suh, H.-W.; Guard, L. M.; Hazari, N. Chem. Sci. 2014, 5, 3859.

[16] Greenhalgh, M. D.; Thomas, S. P. J. Am. Chem. Soc. 2012, 134, 11900.

[17] Fujihara, T.; Xu, T.; Semba, K.; Terao, J.; Tsuji, Y. Angew. Chem. Int. Ed. 2011, 50, 523.

[18] Ohmiya, H.; Tanabe, M.; Sawamura, M. Org. Lett. 2011, 13, 1086.

[19] Fujihara, T.; Tani, Y.; Semba, K.; Terao, J.; Tsuji, Y. Angew. Chem., Int. Ed. 2012, 51, 11487.
[20] Zhang, L.; Cheng, J.; Carry, B.; Hou, Z. J. Am. Chem. Soc. 2012, 134,14314

[21] Correa, A.; Martin, R. J. Am. Chem. Soc. 2009, 131, 15974.

[22] Fujihara, T.; Nogi, K.; Xu, T.; Terao, J.; Tsuji, Y. J. Am. Chem. Soc. 2012, 134, 9106.

[23] Hung, T.-V.; Olafs, D. ACS Catal. 2013, 3, 2417.

[24] Leon, T.; Correa, A.; Martin, R. J. Am. Chem. Soc. 2013, 135, 1221.

[25] Correa, A.; Leon, T.; Martin, R. J. Am. Chem. Soc. 2014, 136, 1062.

[26] Liu, Y.; Cornella, J.; Martin, R. J. Am. Chem. Soc. 2014, 136, 11212.

[27] Moragas, T.; Cornella, J.; Martin, R. J. Am. Chem. Soc. 2014, 136, 17702.

[28] Sayyed, F. B.; Tsuji, Y.; Sakaki, S. Chem. Commun. 2013, 49, 10715.

[29] Sayyed, F. B.; Sakaki, S. Chem. Commun. 2014, 50, 13026.

[30] Wang, X.; Liu, Y.; Martin, R. J. Am. Chem. Soc. 2015, 137, 6476.

[31] Fujihara, T.; Horimoto, Y.; Mizoe, T.; Sayyed, F. B.; Tani, Y.; Terao, J.; Sakaki, S.; Tsuji, Y. Org. Lett. 2014, 16, 4960.

[32] Ostapowicz, T. G.; Schmitz, M.; Krystof, M.; Klankermayer, J.; Leitner, W. Angew. Chem., Int. Ed. 2013, 52, 12119.

[33] Wu, L.; Liu, Q.; Fleischer, I.; Wu, L.; Liu, Q.; Fleischer, I.; Jackstell, R.; Beller, M. Nat. Commun. 2014, 4, 4091.

[34] Wang, W.; Yan, Z. J.; Yuan, Y.; Sun, F. X.; Zhao, M.; Ren, H.; Zhu, G. S. Acta Chim. Sinica 2014, 72, 557. (王维，间卓君，元野，孙福 兴, 赵明, 任浩, 朱广山, 化学学报, 2014, 72, 557.)

[35] Gao, M. Y.; Jiang, D.; Sun, D. K.; Hou, B.; Li, D. B. Acta Chim. Sinica 2014, 72, 1092. (高梦语，姜东，孙德魁，侯博，李德宝，化 学学报, 2014, 72, 1092.)

[36] Li, F.; Suo, Q.; Hong, H.; Zhu, N.; Wang, Y.; Han, L. Chin. J. Org. Chem. 2014, 34, 2172. (李发旺, 索全伶, 洪海龙, 竺宁, 王亚琦, 韩利民, 有机化学, 2014, 34, 2172.)

[37] Zheng, G. D.; Yan, Y.; Cao, X. Z.; Xu, J. Q.; Gao, S.; Tong, S. L.; Gao, D. Acta Chim. Sinica 1995, 53, 299. (郑国栋，阎雁，曹锡章, 徐吉庆, 高赛, 佟珊玲, 高德, 化学学报, 1995, 53, 299.)

[38] He, M.; Sun, Y.; Han, B. Angew. Chem. Int. Ed. 2013, 52, 9620.

[39] Lu, X. B.; Darensbourg, D. J. Chem. Soc. Rev. 2012, 41, 1462.

[40] Sakakura, T.; Choi, J.; Yasuda, H. Chem. Rev. 2007, 107, 2365

[41] He, L.-N. Carbon Dioxide Chemistry, Science Publisher, Beijing, 2013. (二氧化碳化学, 科学出版社, 北京, 2013.)

[42] He, L.-N.; Wang, J.-Q.; Wang, J.-L. Pure Appl. Chem. 2009, 81, 2069.

[43] Liu, A.-H.; Ma, R.; Song, C.; Yu, A.; He, L.-N. Angew. Chem., Int. Ed. 2012, 52, 11306 . 